\title{
Spurenstoffe in der aquatischen Umwelt
}

Organische Spurenstoffe werden im Umweltschutz und im Konsumentenschutz als ein Thema angesehen, dem in Zukunft vermehrt Bedeutung zukommen wird. Dies gilt auch für den Schutz der Ressource Wasser als Lebensraum für aquatische Biozönosen und als Lebensmittel für den Menschen. Zahlreiche internationale Aktivitäten auf wissenschaftlicher, technischer, politischer und legistischer Ebene dokumentieren die zunehmende Beschäftigung mit der Thematik.

Europa und natürlich auch Österreich weisen eine langjährige Tradition im Umgang mit Stoffen auf, welche die Wassergüte negativ beeinflussen und sowohl den aquatischen Lebensraum als auch die Nutzung des Wassers durch den Menschen betreffen. Die in den letzten Jahrzehnten gesetzten Maßnahmen des Gewässerschutzes haben dazu beigetragen, dass unsere Wasserkörper weitestgehend einen guten Immissions-Zustand aufweisen. Dennoch wurde in den letzten Jahren vermehrt die Frage aufgeworfen, inwieweit das Vorhandensein von Stoffen im Mikro- oder Nanogrammbereich negative Auswirkungen auf die Stabilität und Fitness von Ökosystemen haben kann. Durch chemisch-analytische Untersuchungen konnten organische Stoffe im Spurenbereich in einer Vielzahl von Gewässern nachgewiesen werden. Ob und inwieweit dieses Vorhandensein jedoch zu einer wesentlichen Beeinträchtigung der Wasserorganismen führt, ist zurzeit noch nicht geklärt. Dies liegt in erster Linie am Fehlen einer geeigneten Methode zur Charakterisierung eines potenziellen Einflusses. Offensichtlich ist nur, dass diese Substanzen kein „akutes“ Problem verursachen, denn dann würden unsere Gewässer nicht den wissenschaftlich abgesicherten guten Zustand aufweisen. Es wird angenommen, dass hier langfristige Effekte am Wirken sind, deren Mechanismen jedoch noch völlig unklar sind.

Während für traditionelle Fragestellungen des Gewässerschutzes, der Saprobie und der Trophie international kalibrierte Methoden zur Verfügung stehen und es eine breite Erfahrung und Tradition in deren Einsatz gibt, steht die Entwicklung gewässerökologisch aussagekräftiger Methoden für die Bewertung von Spurenstoffen noch am Anfang. Informationen über akut toxische Effekte sind zwar für viele Spurenstoffe bekannt, diese liegen jedoch deutlich über den in der aquatischen Umwelt gefundenen Konzentrationen und sind nicht geeignet, die chronischen Langzeiteffekte zu beurteilen.

Auf die Erkenntnis gestützt, dass die potenziellen Auswirkungen von Spurenstoffen auf chronischen Langzeiteffekten beruhen und nicht auf kurzfristigen akuten Toxizitäten, besteht jedoch die zeitliche Möglichkeit - und Notwendigkeit -, die Thematik auf breiter Ebene sachlich fundiert zu untersuchen und zu diskutieren. Dadurch lassen sich anlassbezogene, kurzfristig entschiedene Reaktionen vermeiden, zumal es für zahlreiche der thematisierten Stoffe bereits Bestimmungen z. B. aus dem Chemikalienrecht gibt. Es ist wichtig, den unterschiedlichen involvierten Fachkreisen, der Politik und den BürgerInnen den Stand der Diskussion, des Wissens aber auch die derzeitigen Wissenslücken zu vermitteln und auf die Notwendigkeit einer wissenschaftlich fundierten Entscheidungsbasis als Grundlage für Managementmaßnahmen hinzuweisen. Die jüngst geführte Diskussion über die Neonicotinoide hat verdeutlicht, wie unabdingbar eine emotionslose, sachliche Diskussion wäre, die es entkoppelt von subjektiven Vorurteilen und reflexartigen Reaktionen erlaubt, Schutzziele zu definieren und deren Einhaltung umzusetzen. Für die Erarbeitung von wissenschaftlichen und gesetzlichen Grundlagen sei den wissenschaftlichen und administrativen Institutionen die dafür notwendige Zeit zugestanden, welche für die Etablierung fundierter neuer methodischer Ansätze benötigt wird. Die internationalen Aktivitäten belegen, dass diese Zeit auch genützt wird, das Verständnis der Thematik voranzutreiben.

In diesem Themenheft der Österreichischen Wasser- und Abfallwirtschaft soll ein Beitrag zu einer öffentlichen und sachlichen Diskussion über Spurenstoffe in der aquatischen Umwelt geleistet werden, indem Begriffe definiert werden, bestehende gesetzliche Rahmenbedingungen erörtert werden, die

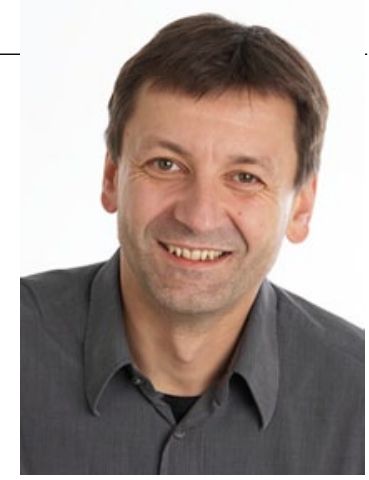

Dr. Norbert Kreuzinger 
Komplexität der Eintragspfade diskutiert wird und die toxikologischen Grundlagen zur Beurteilung von Effekten vorgestellt werden. Die Autoren haben bewusst die Beiträge derart gestaltet, dass einerseits die Komplexität der Thematik verdeutlicht wird, auf der anderen Seite die Inhalte aber auch von fachfremden Personen nachvollzogen werden können. Sie werden in den Beiträgen also keine (Be-) Wertung der Thematik finden, sondern einen umfassenden Überblick über wesentliche thematische Teilbereiche und Diskussionspunkte. Als Leiter des ÖWAV-Arbeitsausschusses „Spurenstoffe“ wünsche ich Ihnen eine spannende und aufschlussreiche Lektüre und uns allen eine fachlich geprägte Auseinandersetzung mit dem Thema der Spurenstoffe in der aquatischen Umwelt!

\section{Dr. Norbert Kreuzinger}

Leiter des ÖWAV-Arbeitsausschusses „Spurenstoffe“

Technische Universität Wien

Institut für Wassergüte, Ressourcenmanagement und Abfallwirtschaft

Karlsplatz 13

1040 Wien

norbkreu@iwag.tuwien.ac.at 